Submitted to ApJ Letters

\title{
A puzzling millisecond pulsar companion in NGC $6266^{1}$
}

\author{
G. Cocozza ${ }^{2,3}$, F. R. Ferraro ${ }^{2}$, A. Possenti ${ }^{4}$, G. Beccari ${ }^{2}$, B. Lanzoni ${ }^{2}$, S. Ranson ${ }^{5}$, R. T. \\ $\operatorname{Rood}^{6}$, N. D'Amico ${ }^{4,7}$
}

\begin{abstract}
We report on the optical identification of the companion to the eclipsing millisecond pulsar PSR J1701-3006B in the globular cluster NGC 6266. A relatively bright star with an anomalous red colour and an optical variability ( $\sim 0.2 \mathrm{mag})$ that nicely correlates with the orbital period of the pulsar ( $\sim 0.144$ days $)$ has been found nearly coincident with the pulsar nominal position. This star is also found to lie within the error box position of an X-ray source detected by Chandra observations, thus supporting the hypothesis that some interaction is occurring between the pulsar wind and the gas streaming off the companion. Although the shape of the optical light curve is suggestive of a tidally deformed star which has nearly completely filled its Roche lobe, the luminosity $\left(\sim 1.9 L_{\odot}\right)$ and the surface temperature $(\sim 6000 \mathrm{~K})$ of the star, deduced from the observed magnitude and colours, would imply a stellar radius significantly larger than the Roche lobe radius. Possible explanations for this apparent inconsistency are discussed.
\end{abstract}

Subject headings: Globular clusters: individual (NGC6266); stars: evolution pulsars: individual (PSR J1701-3006B) - binaries: close.

\footnotetext{
${ }^{1}$ Based on observations with the NASA/ESA HST, obtained at the Space Telescope Science Institute, which is operated by AURA, Inc., under NASA contract NAS5-26555.

${ }^{2}$ Dipartimento di Astronomia Università di Bologna, via Ranzani 1, I-40127 Bologna, Italy,

${ }^{3} \mathrm{INAF}$, Osservatorio Astronomico di Bologna, via Ranzani 1, I-40127 Bologna, Italy

${ }^{4}$ INAF, Osservatorio Astronomico di Cagliari, Loc. Poggio dei Pini, Strada 54, I-09012 Capoterra, Italy

${ }^{5}$ National Astronomy Observatory, 520 Edgemont Road, Charlottesville, VA, 22903, USA

${ }^{6}$ Astronomy Department, University of Virginia, P.O. Box 400325, Charlottesville, VA, 22904, USA

${ }^{7}$ Dipartimento di Fisica Università di Cagliari, Cittadella Universitaria, I-09042 Monserrato, Italy
} 


\section{Introduction}

NGC 6266 (M62) is one of the most massive and brightest $\left(M_{V}=-9.19\right.$; Harris 19961) Galactic Globular Clusters (GCs), also is characterized by high values of the central density $\left(\log \rho_{0} \sim 5.47\right.$, with $\rho_{0}$ in units of $M_{\odot} \mathrm{pc}^{-3}$; Beccari et al. 2006). It displays a King-model density profile with an extended core $\left(\sim 19^{\prime \prime}\right)$ and a modest value of the concentration parameter $(c=1.5$; Beccari et al. 2006).

Six binary millisecond pulsars (MSPs) have been discovered in M62 (D'Amico et al. 2001; Jacoby et al. 2002; Possenti et al. 2003, hereafter P03) and it ranks fifth of the GCs in wealth of MSPs, after Terzan 5, 47 Tucanae, M15 and M282. P03 presented phaseconnected timing solutions, yielding precise celestial coordinates, for three of the MSPs in NGC 6266. One of them, PSR J1701-3006B (hereafter PSR 6266B) displays partial or total eclipses of the radio signal at $1.4 \mathrm{GHz}$ near its superior conjunction (in the convection adopted throughout this paper, this corresponds to orbital phase $\phi=0.25$ ), clearly due to gas streaming off the companion. The pulsar orbit is circular, with a projected semimajor axis of only $\sim 0.11 R_{\odot}$. P03 suggested two options for explaining the behaviour of the PSR 6266B system. The first option is that the pulsar companion is a non-degenerate bloated star, whose mass loss is sustained by ablation of its loosely bound surface layers by the relativistic wind emitted by the pulsar. In this case, PSR 6266B may resemble PSRs B1957+20 (Fruchter et al. 1990) and J2051-0827 (Stappers et al. 2001): the optical light curve of their companion star presents a maximum when the side of the companion facing the pulsar points toward the observer (i.e., at the pulsar inferior conjunction: $\phi=0.75$ ). This is a clear signature of the irradiation of the companion surface by the pulsar flux. The second option has the pulsar companion as a tidally deformed star overflowing its Roche lobe due to the internal nuclear evolution. In this case, the system may be more like PSR J1740-5340 (D'Amico et al. 2001; Ferraro et al. 2001), where irradiation effects are negligible (Orosz \& van Kerkwijk 2003) and the optical light curve of the companion is dominated by ellipsoidal variations (Ferraro et al. 2001), i.e. it shows two maxima at quadratures $(\phi=0.0$ and 0.5$)$.

We present the optical identification of the companion to PSR 6266B, based on highquality phase-resolved photometry obtained with the Hubble Space Telescope (HST), and X-ray emission detected with Chandra .

\footnotetext{
${ }^{1}$ Everywhere in this paper, we refer to the data at http://www.physics.mcmaster.ca/Globular.html

${ }^{2}$ See http://www.naic.edu/ pfreire/GCpsr.html for a catalog of MSPs in GCs with relative references
} 


\section{Observations and data analysis}

HST OBSERVATIONS. The photometric data presented here consist of a set of highresolution images obtained on August 12004 by using the Advanced Camera for Survey (ACS), on-board the HST, and retrieved from the ESO/ST-ECF Science Archive. The data comprise three $R$-band exposures $(2 \times 340 \mathrm{~s}$ and $1 \times 30 \mathrm{~s}$ exposures $)$, two $B$-band exposures (of 120 and $340 \mathrm{~s}$ ) and four $H_{\alpha}$-band exposures (of 340,1050, 1125, 1095s). The three longest exposures in $H_{\alpha}$ are the combination of three sub-exposures. An additional set of Wide Field Planetary Camera 2 (WFPC2, Prop. 10845, PI:Ferraro) data has been secured through the $H_{\alpha}$ filter with the specific aim of testing the possible variability of the companion:

ten 31200 s exposures were taken between May 2 and May 5 2007. In order to best resolve stars in the most crowded regions, the Planetary Camera (with a resolution of $0.046^{\prime \prime} /$ pixel) of the WFPC2 was pointed at the cluster center. The photometric analysis of the ACS dataset has been carried out using the ACS module of the Dolphot4 package (Dolphin 2000), using the FLT.fits datasets for the photometry, and the drizzled images as references for the geometric distortion correction. We set the photometry parameters as recommended in the Dolphot manual, obtaining a final catalog of $B, R$ and $H_{\alpha}$ magnitudes, calibrated according to Sirianni et al. (2005). The final catalog is reported to an absolute astrometric system by cross-correlating the stars in common with the data set of Beccari et al. (2006), which has been astrometrized by using the standard stars from the new Guide Star Catalog II (GSCII) lying in the considered field of view (FoV). At the end of the procedure, the rms residuals were of the order of $\sim 0.5^{\prime \prime}$ both in RA and Dec; we assume this value as representative of the astrometric accuracy.

In order to carefully search for any variability from the MSP companion, we reanalyzed the $H_{\alpha}$ images in a small area around the nominal position of PSR 6266B by using ROMAFOT (Buonanno et al. 1983). This package has been specifically developed to perform accurate photometry in crowded fields, and it also allows a visual inspection of the quality of the PSF-fitting procedure. In particular, we extracted $50 \times 50$-pixels $\left(\sim 2.5^{\prime \prime} \times 2.5^{\prime \prime}\right)$ sub-images from the ten original $H_{\alpha}$ ACS frames, each centered on the nominal position of PSR 6266B. In order to optimize the detection of faint objects, we performed the search procedure on the median image. Then, we adopted the resulting mask with the star positions to the individual sub-images, and performed the PSF-fitting procedure separately on each of them. We adopted the same strategy for the analysis of the nine WFPC2 $H_{\alpha}$ images,

\footnotetext{
${ }^{3}$ Only 9 exposures have been used in the analysis since one was made useless by a cosmic ray passage.

${ }^{4}$ The DOLPHOT packages, including the ACS module and all the documentation, can be found at http://purcell.as.arizona.edu/dolphot/.
} 
after the careful alignement with the previously astrometrized ACS frames. The resulting instrumental magnitudes were transformed to a common system, and we compiled a final catalog with coordinates and magnitudes for all the stars identified in the considered 19 sub-images. The photometric calibration of the instrumental magnitudes and the absolute celestial coordinates were determined by using all the stars in common with the overall ACS catalog, calibrated and astrometrized as discussed above.

Chandra Observations. NGC 6266 was observed for $63 \mathrm{ks}$ on May 2002 with the Chandra Advanced CCD Imaging Spectrometer (ACIS). The ACIS-S3 chip was pointed at the cluster center, its FoV $\left(8^{\prime} \times 8^{\prime}\right)$ entirely covering the half-mass radius (1.23'; Harris 1996). The data were reduced using the CIAO software (v. 3.3.0), reprocessing the level 1 event files; the task acis_run_hotpix was used to generate a new badpixel file. Then we applied the corrections for pixels randomization, good time intervals and grades. Our analysis includes only photons with ASCA grades of $0,2,3,4$ and 6 . About $1.5 \mathrm{ks}$ of observations were not considered in the analysis because of the high background level. We searched for discrete sources in our level 2 event file using Wavdetect (Freeman et al. 2002), performing the source detection separetely on the $0.2-1,1-2,2-8$, and $0.2-8 \mathrm{keV}$ bands. The detection threshold

was set to $10^{-5}$, and the source detection was performed increasing the sequence of wavelets from 1 to 16 by factors of $\sqrt{2}$. More than 110 sources were detected in the $0.2-8 \mathrm{keV}$ band in the entire chip. The astrometry of all the detected sources was corrected by using the Aspect Calculator provided by the Chandra X-Ray Center. Then we searched for possible optical counterparts both in our HST images and catalog. About 5 bright stars and 2 faint and extended objects (likely background galaxies) were found to coincide with the X-ray sources well outside the half-mass radius, where the stellar density is relatively low. We therefore used these optical counterparts for extending the astrometric solution of HST to the Chandra sources, thus obtaining the same accuracy in the absolute astrometry for both the optical and the X-ray sources.

\section{Results}

Figure 1 shows the $3^{\prime \prime} \times 3^{\prime \prime}$ WFPC2 $H_{\alpha}$ map centered on the PSR 6266B nominal position (marked with a cross), as derived from timing (see P03). An accurate photometric analysis has been carried out for all the $\sim 30$ stars found in our catalog within a distance of $1.5^{\prime \prime}$ from the PSR 6266B position. This correspond to 3 times the $1 \sigma$ uncertainty $\left(0.5^{\prime \prime}\right)$ in the absolute astrometry of the HST data. In particular, we have extracted 19 individual images from our dataset (10 exposures with ACS and 9 with WFPC2) and compared the resulting magnitudes for each object in order to search for variability and estimate the typical 
photometric uncertainties at different magnitude levels. Only one source in the catalog (hereafter named COM6266B, marked with a small circle in Fig. 1) showed $H_{\alpha}$ variability $\left(\Delta H_{\alpha} \sim 0.2 \mathrm{mag}\right)$ significantly larger than the typical $\mathrm{rms}$ magnitude fluctuations of stars of similar luminosity ( $\delta H_{\alpha} \sim 0.02 \mathrm{mag}$ in ACS data, and $\delta H_{\alpha} \sim 0.04 \mathrm{mag}$ in WFPC2 data). Its celestial coordinates are $\alpha_{\mathrm{J} 2000}=17^{\mathrm{h}} 01^{\mathrm{m}} 12^{\mathrm{s}} .690$ and $\delta_{\mathrm{J} 2000}=-30^{\circ} 06^{\prime} 48^{\prime \prime} .61$, whereas the mean apparent magnitudes are $B=20.58, V=19.48, R=19.02, H_{\alpha}=18.65$ ( $V$ is from Beccari et al. 2006). It is located at $0.5^{\prime \prime}$ from PSR 6266B just at the edge of its positional error circle.

Figure 2 reports the $(R, H \alpha-R)$ and $(R, B-R)$ Color Magnitude Diagrams (CMDs) for all the stars detected in the $20^{\prime \prime} \times 20^{\prime \prime}$ box centered on the PSR $6266 \mathrm{~B}$ nominal position. COM 6266B is marked with a large filled triangle in both the CMDs. As apparent from the figure, the star has almost the same luminosity of the cluster Turn Off, but it shows an anomalous red color which locates it out of the Main Sequence. The photometric properties imply that it is not a degenerate star and that it has a moderate $H_{\alpha}$ excess, indicating the presence of ionized gas surrounding the system. These findings are consistent with the irregulaties seen in the radio signal of PSR 6266B and supports the scenario that COM 6266B is a mass losing star.

In order to firmly assess the physical connection between COM 6266B and the binary pulsar PSR 6266B, we have accurately investigated the time scale of the optical variability 5 (in the 0-1 day range). The $H_{\alpha}$ time series (19 points) was processed using the GRATIS $\chi^{2}$ fitting routine, a code developed at the Bologna Astronomical Observatory in order to study the periodicity of variable stars (see, e.g., Clementini et al. 2000). Following the procedure fully described in Ferraro et al. (2001), the most significant (99\% confidence interval) 6 periodicity was found at a period of $0.1446 \pm 0.0015$ day, consistent, within the uncertainties, with the orbital period of PSR 6266B derived from timing $\left(P_{b}=0.1445454303(6)\right.$ day, where the figure in parenthesis is the uncertainty, at $99 \%$ confidence level, on the last quoted digit; P03). Given that, we have folded the 19 magnitude values, by using $P_{b}$ and the reference epoch of the PSR 6266B radio ephemeris $\left(T_{0}=52047.258199(2)\right.$; P03). The results are shown in Fig. 3 . As apparent, the ACS and the WFPC2 data (obtained about 3 years later)

\footnotetext{
${ }^{5}$ Note that COM $6266 \mathrm{~B}$ has a close companion located at only 4 pixels $\left(\sim 00^{\prime \prime} 19\right)$ West in the ACS map (see Fig 1). This object turns out to be a normal Sub-Giant Branch star, slightly brighter $(\sim 0.5$ mag in $B, R$ and $H_{\alpha}$ ) than COM 6266B and not displaying any evidence of variability. Although very close to each other, the two stars are clearly separable in the high resolution ACS and WFPC2 images and we have verified that the photometric analysis of COM 6266B is not affected by the presence of this close star.

${ }^{6}$ The significance level has been obtained from the reduced $\chi^{2}$ of the Fourier time series fit (at different modulation periods) to the $H_{\alpha}$ data (see for example Figure 2 of Ferraro et al. 2001).
} 
agree with each other, drawing a well defined light curve with two distinct maxima at about $\phi=0.0$ and $\phi=0.5$. This fact, as well as the phases and the amplitudes of the minima, confirms that the optical modulation is associated with the pulsar binary motion and strongly suggests that COM 6266B is a deformed star overflowing its Roche lobe (see $§ 1$ ).

Additional properties of this system can be derived from the analysis of the Chandra data. We found an X-ray source located at only $\sim 0$ 0.4 from COM 6266B and at 0..3 from the nominal radio position of PSR 6266B. The derived coordinates of the X-ray source are $\alpha_{\mathrm{J} 2000}=17^{\mathrm{h}} 01^{\mathrm{m}} 12^{\mathrm{s}} .700$ and $\delta_{\mathrm{J} 2000}=-30^{\circ} 06^{\prime} 49^{\prime \prime} 08$, and the $1 \sigma$ uncertainty area on its position is encircled in Fig.1 (dashed line). Given the number of sources (50) detected within the half-mass radius of NGC6266, the probability of a chance superposition of a X-ray source within $00^{\prime \prime} 3$ from the radio position of PSR 6266B is $\sim 7 \times 10^{-4}$, strongly supporting the association of the detected source with the PSR 6266B system. The (background substracted) photons counts are $18.3_{-4.2}^{+5.3}$ (soft: $0.2-1 \mathrm{keV}$ ), 23.7 $7_{-4.9}^{+6.0}$ (medium: $1-2 \mathrm{keV}$ ) and $9.8_{-3.1}^{+4.3}$ (hard: $2-8 \mathrm{keV}$ ), implying HR1 1.3 and $H R 2 \simeq 0.4$ (where HR1=medium/soft counts and HR2=hard/medium counts). Hence the source is harder than the typical MSP population observed in GCs (e.g. Grindlay et al. 2002) and the HR1, HR2 values resemble those of a source in which the pulsar wind shocks the material released from the companion (see, e.g., the cases of PSR J1740-5340 in NGC 6397, and PSR J0024-7204W in 47Tuc; Grindlay et al. 2002, and Bogdanov et al. 2005, respectively). Even if the small number of photons prevents a detailed spectral analysis, for an absorption column density $N_{H}=2.2 \times 10^{21} \mathrm{~cm}^{-2}$ (Pooley et al. 2003), the counts in each band and the HR1 and HR2 values are consistent 7 with a power law model having a photon index $\simeq 2.5$, and a total unabsorbed flux $F_{X} \sim 10^{-14} \mathrm{erg} \mathrm{cm}^{-2} \mathrm{~s}^{-1}$ in the $0.2-8 \mathrm{keV}$ band, translating (for a distance of $6.6 \mathrm{kpc}$; Beccari et al. 2006) into a X-ray luminosity of $L_{X} \sim 6 \times 10^{31} \mathrm{erg} \mathrm{s}^{-1}$.

\section{Discussion}

Many observed features of COM 6266B (namely, its anomalous red colour, the $H_{\alpha}$ excess, the shape of the light curve, the X-ray emission from the binary) are suggestive of a tidally deformed star which is experiencing heavy mass loss, similar to the system found in NGC 6397 by Ferraro et al. (2001). On the other hand, other photometric properties of the source do not fit this picture, as described below.

By assuming the reddening $E(B-V) \sim 0.47$ and the distance quoted by Beccari et al. (2006), and adopting $[\mathrm{Fe} / \mathrm{H}]=-1.1$ and an age of 12 Gyr for NGC 6266, we have determined

\footnotetext{
${ }^{7}$ The PIMMS tool has been used for these estimates: http://heasarc.gsfc.nasa.gov/Tools/w3pimms.html
} 
the physical parameters of COM 6266B from the comparison of its position on the CMDs (Fig.2) with the isochrones of Pietrinferni et al. (2004). The resulting effective temperature, bolometric luminosity and radius of this star are $T_{\text {eff }} \sim 6000 \pm 500 \mathrm{~K}, L_{\text {bol }} \sim 1.9 \pm 0.2 L_{\odot}$, and $R \sim 1.2 \pm 0.2 R_{\odot}$, respectively, where the quoted uncertainties are conservative estimates, essentially due to the uncertanties in the distance modulus $( \pm 0.15 \mathrm{mag})$ and in the reddening $( \pm 0.05 \mathrm{mag})$. If the luminosity variations shown in Fig. 3 are mainly due to tidal deformations of the companion star, the stellar radius $R$ is expected to be of the order of the Roche lobe radius $R_{L}$. The latter can be estimated from the mass function and the projected semi-major axis of PSR 6266B (derived from pulsar timing; P03), provided that a pulsar mass $M_{\mathrm{psr}}$ and an orbital inclination $i$ are assumed. As already discussed in P03, the presence of radio eclipses indicates that $i$ is not small. Conservatively taking $i \gtrsim 20^{\circ}$ and assuming $M_{\mathrm{psr}}=1.4 M_{\odot}$, the companion star is expected to span a mass interval ranging from $0.125 M_{\odot}\left(i=90^{\circ}\right)$ to $0.41 M_{\odot}\left(i=20^{\circ}\right) 8$, corresponding to $R_{L} \sim 0.26 R_{\odot}$ and $0.40 R_{\odot}$, respectively. The value of $R_{L}$ is only slightly affected by the choice of $M_{\mathrm{psr}}$, being, for instance, $R_{L} \lesssim 0.45 R_{\odot}$ for $0.7 M_{\odot} \leq M_{\mathrm{psr}} \leq 3.0 M_{\odot}$ (a safely large neutron star mass range; see, e.g., Freire et al. 2008). Note that in order to match the estimated $R_{L}$ with the observed $R$ it should be $i \sim 3^{\circ}$ (for $M_{\mathrm{psr}}=1.4 M_{\odot}$ ), thus implying an unreliable companion mass of $\sim 6 M_{\odot}$. In summary, for all plausible binary parameters, it turns out that the observed stellar radius of COM 6266B is significantly larger $\left(R \gtrsim 3 R_{L}\right)$ than the expected Roche lobe radius of the companion to PSR 6266B, i.e. COM 6266B appears $\gtrsim 10$ times brighter than the expected luminosity of any pulsar companion having an effective temperature $T_{e}=6000 \mathrm{~K}$.

Under the hypothesis that COM 6266B is the companion to PSR 6266B, what is the origin of this remarkable discrepancy? A number of possibilities are examined below.

(i) The binary system could not belong to the cluster. In this case the adopted distance would be inappropriate and the value of the radius deduced from the luminosity would be largely overestimated. On the other hand, the celestial position of PSR 6266B is at less than $2^{\prime \prime}$ from the cluster center, and the pulsar shows a negative value of the spin period derivative, as expected if PSR 6266B lies in the cluster potential well.

(ii) The optical luminosity of COM 6266B could be dominated by strong non-thermal processes, possibly triggered by the pulsar spin down power $L_{\text {sd }} \lesssim 10 L_{\odot}$ (see P03). Also in this case the value of the stellar radius deduced from the observed luminosity could be significantly overestimated. However, At the orbital separation of $1.4 R_{\odot}$, the energy captured by a Roche lobe filling companion would be of the order of $L \sim 0.1-0.2 L_{\odot}$ which

\footnotetext{
${ }^{8}$ Note that in both cases the orbital separation of the system turns out to be $a \sim 1.4 R_{\odot}$.
} 
is too low to explain the observed value. Moreover, it is hard to explain how a large nonthermal component may generate a light curve with maxima that fall at orbital phases nicely consistent with those expected for light curves modulated by ellipsoidal variations.

(iii) COM 6266B could be the blend of two low-luminosity stars: one star would be the optically variable companion to PSR $6266 \mathrm{~B}$ and the other one a non-variable star in the foreground (background). While stellar blends are not uncommon in the highly crowded GC cores, this possibility only partially alleviates the problem. In fact, by assuming that COM 6266B is the blend of two stars of equal mean $H_{\alpha}$ magnitude (i.e., $H_{\alpha}=19.35$ ), the observed ellipsoidal modulation could only be produced if the pulsar companion varies by $\Delta H_{\alpha} \sim 0.35 \mathrm{mag}$, which is a rather extreme value for variabilities induced by tidal distortions. Moreover, even in this case, the star would have a radius $R \gtrsim 1.7 R_{L}$. Matching $R$ with $R_{L}$ would require that COM $6266 \mathrm{~B}$ is e.g. the blend of a non-variable star with $H_{\alpha}=18.7$ and a star with mean magnitude $H_{\alpha} \sim 21$ varying by $\Delta H \alpha>3$ mag. We note that similar considerations would apply also if PSR 6266B were a triple system.

In conclusion, while option (iii) seems to be a quite unlike ad hoc scenario, options (i) and (ii) will be finally addressed as soon as spectroscopic data are available for the system. This will allow us to investigate the nature (thermal or not thermal) of the optical emission and the association of the source to the cluster (via the determination of the radial velocity), hence shedding some light on the nature of this puzzling object.

This research was supported by the Agenzia Spaziale Italiana (ASI I/088/06/0), PRININAF2006 and by the Ministero dell'Istruzione, dell'Università e della Ricerca, and it is part of the Progetti Strategici di Ateneo 2006 granted by University of Bologna. AP and NDA

also acknowledge financial support from PRIN-MIUR2005. We thank the Referee for the careful reading of the manuscript.

\section{REFERENCES}

Beccari, G., Ferraro, F. R., Possenti, A., Valenti, E., Origlia, L., \& Rood, R. T., 2006, AJ, 131, 2551

Bogdanov, S., Grindlay, J. E. and van den Berg, M., 2005, ApJ, 630, 1029

Buonanno, R., Buscema, G., Corsi, C. E, Ferraro, I., \& Iannicola, G., 1983, A\&A, 126, 278

Clementini, et al., 2000, AJ, 120, 2054 
D’Amico, N., Lyne, A. G.,Manchester, R. N., Possenti, A., \& Camilo, F., 2001, ApJ, 561, L171

Dolphin, A. E., 2002, PASP, 112, 1383

Ferraro, F. R., Possenti, A., D’Amico, N., \& Sabbi, E., 2001, ApJ, 561, L93

Freeman, P. E., Kashyap, V., Rosner, R., \& Lamb, D. Q., 2002, ApJS, 138, 185

Freire, P.C.C., Wolszczan, A., van den Berg, M., \& Hessels, J. W. T., 2008, ApJ, in press

Fruchter, A. S. et al., 1990, ApJ, 351, 642

Grindlay, J. E., Camilo, F., Heinke, C. O., Edmonds, P. D., Cohn, H. \& Lugger, P., 2002, ApJ, 581, 470

Harris, W. E., 1996, AJ, 112, 1487

Jacoby, B. A., Chandler, A. M., Backer, D. C. Anderson, S. B., \& Kulkarni, S. R., IAUC 7783,2002

Orosz, J. A., \& van Kerkwijk, M. H., 2003, A\&A, 397, 237

Pietrinferni, A., Cassisi, S., Salaris, M., \& Castelli, F., 2004, ApJ, 612, 168

Pooley, D., et al., 2003, ApJ, 591, L131

Possenti, A., D’Amico, N., Manchester, R. N., Camilo, F.,Lyne, A. G., Sarkissian, J., Corongiu, A., 2003, ApJ, 599, 475 (P03)

Sirianni, et al., 2005, PASP, 117, 1049

Stappers, B. W., van Kerkwijk, M. H., Bell, J. F., \& Kulkarni, S. R., 2001, ApJ, 548, L183

This preprint was prepared with the AAS LATEX macros v5.2. 


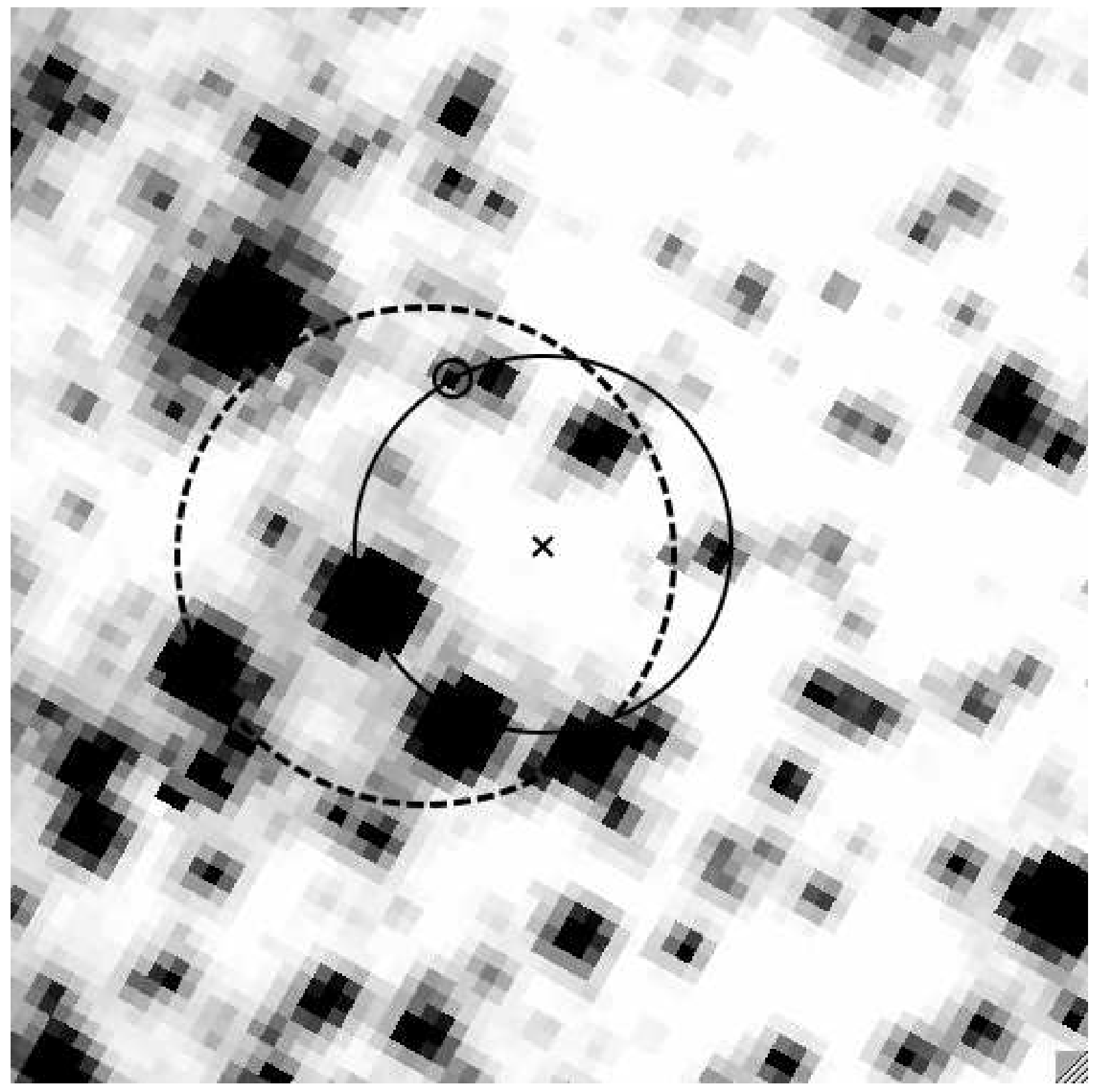

Fig. 1.- Finding chart for COM J1701-3006B showing the median-combined WFPC2 $H_{\alpha}$ image. The region covers $3^{\prime \prime} \times 3^{\prime \prime}$; the cross marks the nominal position of PSR 6266B, with the $1 \sigma$ error circle $\left(0.5^{\prime \prime}\right.$, solid line). The smaller circle shows the optical counterpart, whereas the dashed line represents the $1 \sigma$ circle $\left(0.7^{\prime \prime}\right)$ in the Chandra position. North is up and East is to the left. 


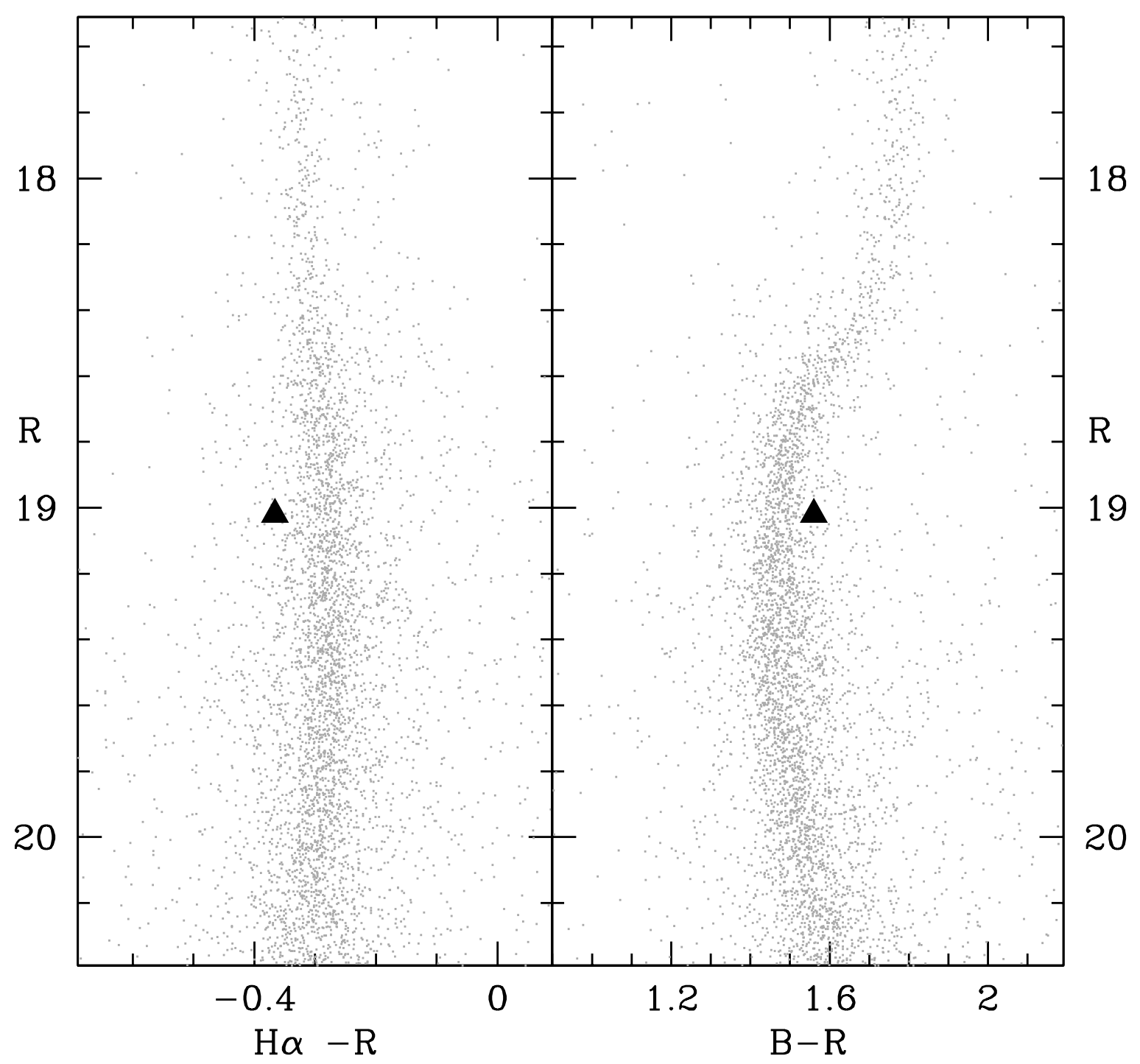

Fig. 2.- $\left(R, H_{\alpha}-R\right)$ and $(R, B-R)$ CMDs for stars identified in a region of $20^{\prime \prime} \times$ $20^{\prime \prime}$ centered at the nominal position of PSR 6266B. The optical counterpart to the pulsar companion COM 6266B is marked with a large filled triangle. 


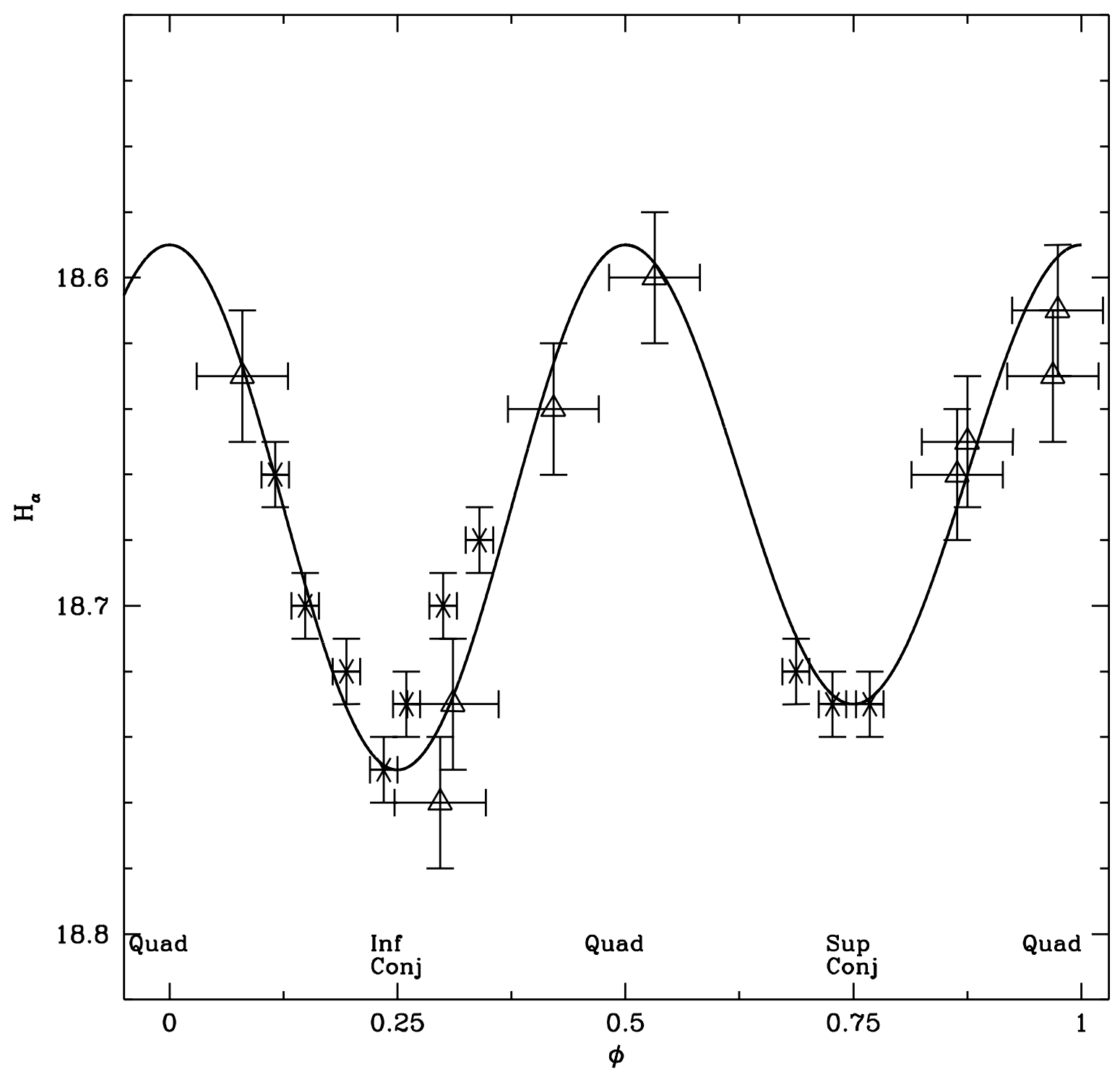

Fig. 3.- $H_{\alpha}$ light curve of COM $6266 \mathrm{~B}$ obtained by using the period and the reference epoch of the radio ephemeris of PSR 6266B. Asterisks represent ACS archive observations performed in August 2004; large empty triangles are the WFPC2 data collected in May 2007. The phases of quadrature and conjunction of COM 6266B are reported for clarity. The solid line represents the Fourier time series (trucated to the second harmonic) that best fits the data. 